\title{
Implementation of virtual clinics for medical retina patients in a \\ tertiary eye care referral centre
}

Karsten Kortuem ${ }^{1,2}$, Katrin Fasler ${ }^{1,3}$, Amanda Charnley ${ }^{1}$, Hussain Khambati ${ }^{1}$, Sandro Fasolo ${ }^{1}$, Menachem Katz ${ }^{1}$, Konstantinos Balaskas ${ }^{1,4}$, Ranjan Rajendram ${ }^{1,5}$, Robin Hamilton ${ }^{1,5}$, Pearse A. Keane ${ }^{1,5}$, Dawn A. Sim ${ }^{1,5}$

${ }^{1}=$ Moorfields Eye Hospital, London, United Kingdom

${ }^{2}=$ University Eye Hospital Munich, Germany

${ }^{3}=$ University Hospital Zurich, Department of Ophthalmology, Zurich, Switzerland

${ }^{4}=$ University of Manchester, School of Biological Sciences, Manchester, UK

5 = National Institute for Health and Research (NIHR) Biomedical Center at Moorfields Eye Hospital and University College of London (UCL) Institute of Ophthalmology, London, UK.

This research received no specific grant from any funding agency in the public, commercial or notfor-profit sectors

The authors declare not to have any competing interest in this study.

\section{Corresponding author:}

Dawn A. Sim

Moorfields Eye Hospital, London, United Kingdom

162 City Road

EC1V 2PD London

dawn.sim@moorfields.nhs.uk 


\section{Precis}

Virtual clinics are a logical solution to overcome the burden of increasing referrals. We describe the implementation of virtual medical retina clinics and reports on the outcomes of the first 1729 patients seen.

\section{Abstract}

\section{Background}

The increasing incidence of medical retinal diseases has created capacity issues across the United Kingdom. In this study, we describe the implementation and outcomes of virtual medical retina clinics (VMRC) at Moorfields Eye Hospital, South Division, London. It represents a promising solution to ensure that patients are seen and treated in a timely fashion

\section{Methods}

First attendances in the VMRC (September 2016 to May 2017) were included. It was open to nonurgent external referrals and to existing patients in a face-to-face clinic (F2FC). All patients received visual acuity testing, dilated fundus photography and OCT scans. Grading was performed by consultants, fellows and allied health care professionals. Outcomes of these virtual consultations and reasons for F2FC referrals were assessed.

\section{Results}

A total number of 1729 patients were included (1543 were internal and 186 external referrals). The majority were diagnosed with diabetic retinopathy $(75.1 \%$ of internal and $46.8 \%$ of external referrals). Of the internal referrals, $14.6 \%$ were discharged, $54.5 \%$ continued in VMRC, and $30.9 \%$ were brought to a F2FC. Of the external referrals, $45.5 \%$ were discharged, $37.1 \%$ continued in VMRC and $17.4 \%$ were brought to a F2FC. The main reason for F2FC referrals was image quality (34.7\%), followed by detection of potentially treatable disease (20.2\%).

\section{Conclusion}

VMRC can be implemented successfully utilising existing resources within a hospital eye service. It may also serve as a first-line rapid-access clinic for low-risk referrals. This would enable medical retinal services to cope with increasing demand and efficiently allocate resources to those who require treatment. 


\section{Introduction}

The origins of the virtual consultation emerged in the early 1900s, when radio communication was invented and "radio doctors" could attend to patients by the means of two-way radios; akin to walkie-talkies of today. However, it was not until the 1940s when the first store-and-forward model of telemedicine transpired when radiology images were transmitted via telephone lines[1]. In the past 10 years, the disruptive impact that optical coherence tomography (OCT) has had on the management of retinal disease has led to a surge in ocular imaging within this subspecialty[2]. This shift in the way we manage retinal disease has lent itself very well to the field of telemedicine.

A major challenge in eye care is capacity: Hospitals continue to receive an increasing number of referrals from community care providers due largely to an aging population and the wider availability of ophthalmic imaging devices in optometry practices[3]. The first published reports on the use of telemedicine to improve efficiency of eye clinics in the United Kingdom (UK) have been in glaucoma [4 5]. These appointments usually consist of non-mydriatic photos of the optic disc, visual fields, and visual acuity measurements carried out by a technician, stored, and later reviewed by an ophthalmologist. These early reports observed that the majority of patients (62\%-89\%) did not require treatment [4], and further demonstrated that virtual clinics were reliable, cost-effective, freeing up clinical appointments for those in need of hospital care[6].

The increase in demand for hospital eye service appointments is felt not only by the glaucoma service but in all other subspecialties of ophthalmology. In 2016, the Royal College of Ophthalmologists (RCOphth) estimated that about 20 patients per month face severe vision loss whilst waiting for their appointment [7]. The situation in the UK is aggravated by one of the lowest numbers of ophthalmologists per capita (52/one million inhabitants) of all industrialized countries[8]. This supply-demand imbalance is also evident globally[9]. To overcome this burden, efforts are underway to improve efficiency, and better utilisation of non-medical staff for the provision of eye care. One example in the UK is the introduction of nurse-led injection clinics, which have been shown to be as safe and acceptable to patients as doctor-led injection clinics[10].

Within medical retina services, projections for the volume of patients with diabetes requiring a hospital appointment suggest we have only hit the tip of the iceberg[11]. In 2012, there were 28 million people with vision-threatening diabetic retinopathy world-wide, estimated to rise to 43 million by 2035[12]. Moreover, recent major advances in the treatment of diabetic retinopathy (DR) such as intravitreal injection therapy, have added to the increasing demands on healthcare systems [13 14].

In the UK a successful and cost-effective national diabetic retinopathy screening services (DRSS) has been existing for more than a decade, and is widely credited with the successful reduction of vision loss from diabetic eye disease in the working age group nationally [15-17]. Early detection of diabetic retinopathy by screening saves patients from having to attend hospital eye services for their annual eye examination. However, the flip side of early detection is the low thresholds for referral set within the screening services. "Referrable retinopathy" does not equate "treatable retinopathy". Hospitals are therefore faced with an increasing number of referrals that do not yet, and may never require treatment.

In this study, we report on the implementation and integration of virtual medical retina clinics from 4 sites of Moorfields Eye Hospital, South Division, London, and report on the outcomes of those consultations. 


\section{Materials \& Methods}

All first attendances (September 2016-May 2017) at the virtual medical retina clinic at Moorfields Eye Hospital, South Division (St. George's hospital, The Nelson Health Centre, Purley War Memorial Hospital and Croydon University Hospital) were included. Patients were either referred internally from a medical retina clinic or new referrals from DRSS. Retrospective clinical data from MR clinics 3 months prior to the next clinic date at the above locations was reviewed over a period of 12 months, more than 7000 patients were scrutinized for suitability for virtual clinics in this 9 months period. This study was part of a registered audit with the Audit Department of Moorfields Eye Hospital NHS trust. Patients who were graded as low risk were referred to the virtual clinic. Risk stratification, inclusion, and exclusion criteria for virtual clinics are listed in supplementary table 1.

The virtual clinic appointment consisted of a clinical examination where past medical history, visual acuity (VA), and non-contact intra-ocular pressure (IOP) were taken by trained nurses and entered in an electronic medical record (EMR), which was, depending on the site, either Medisoft (Medisoft Ltd., Leeds, United Kingdom) or OpenEyes (OpenEyes Foundation, London, United Kingdom). Patients then received dilated fundus photography. Depending on the availability of retinal imaging at locations, either 2-field $45^{\circ}$ fundus photography or wide field fundus photography (Optos) were obtained[18]. Additionally, every patient received an OCT volume scan (Topcon 3D OCT scan)[19].

An intranet-based worklist with data from EMRs and patient administration system (Silverlink PAS) was created using Microsoft SQL Server Reports Software (Microsoft Corporation). Patients were identified from this tool by reviewers. In total, there were 6 reviewers; three were ophthalmological consultants, one medical retinal fellow, one optometrist and one a senior screener grader with a DRSS background. For diabetic patients, grading of findings was performed according to the national UK guidelines[20]. According to these, retinopathies are graded into four levels: none (RO), background (R1), pre-proliferative (R2), proliferative (R3). Maculopathy and photocoagulation are graded as absent (M0, P0) or present (M1, P1). The review took place within one week of examination. After finishing the virtual review, a letter with the outcome was sent to the patient, the GP, and if applicable the DRSS. Outcome could be either follow-up in the virtual clinic, follow-up in a face to face clinic or discharge. Period of observation was from June 2016 until June 2017.

Data was extracted into an Excel spreadsheet from the hospital's data warehouse containing PAS and EMR data, running on a Microsoft SQL server in the hospital's data centre. All patient encounters were reviewed manually in the EMRs to include the disease state and the outcome. Outcomes were stratified by follow-up in virtual clinic, discharge from virtual clinic and referral to face to face medical retina clinic. Face to face referrals were further categorized by:

- Urgent referral needed, i.e. urgent need for an intervention like pan-retinal laser treatment in proliferative DR

- Routine referral e.g. due to cataract or non-urgent interventions needed

- Patient request as noted by nurses or technicians

- Not suitable for virtual clinic, e.g. patient is wheelchair bound

- Booking error. That patient was not intended to turn into a virtual consultation

- Glaucoma suspect. On assessment, there was suspicion of glaucoma, when not noted before in the records

- Image quality. In the presence of optical axis opacity or inadequate photographs, patients were referred to face to face consultation.

Statistical analyses were performed using SPSS 24 (IBM Corporation) for Microsoft Windows. 
Results

A total of 1729 patients were referred to the virtual clinic. The average time for internal referrals between last consultation in a medical face-to-face clinic and their appointment in the virtual clinic was 107.4 days (SD +/- 84.3 days). The average waiting time for new referrals was 45.3 days (SD +/27.6 days). Table 1 shows the patient demographics, the referral source of all referred patients, and the rate of patients who did not attend their first appointment.

\section{Diagnoses of patients seen in virtual medical retina clinic}

$75.1 \%$ (internal referrals) respectively $46.8 \%$ (external referrals) of patients were reviewed for diabetic eye disease, with $28.6 \%$ (internal referrals)/20.4\%(external referrals) mild non-proliferative diabetic retinopathy (R1M1 equivalent), $22.8 \%$ (internal referrals)/24\% (external referrals) previously treated stable retinopathy (R3S equivalent), 40\% (internal referrals)/20.4\% (external referrals) previously treated stable maculopathy (M1S equivalent). Age-related macular degeneration (AMD), retinal vein occlusion (RVO), and central serous retinopathy (CSR) were the most common diagnoses in descending order after diabetes. Table 2 gives an overview of the medical data of all patients who attended their first appointment at the virtual medical retina clinic.

\section{Outcome of virtual medical retina consultations}

Table 2 also indicates what the outcomes (continue in virtual clinic, bring patient to face to face clinic or discharge) of those patients were. Of all internal referrals, $14.6 \%$ of patients were discharged, $54.5 \%$ of patients could continue their therapy in a virtual clinic and $30.9 \%$ of patients were booked for a face-to-face appointment. For new referrals, $45.5 \%$ of patients could be discharged straight away, $37.1 \%$ of patients continued virtually and $17.4 \%$ of patients were brought to a face-to-face clinic. The decisions made in correlation to the clinical rank of the reviewing person can be seen in table 4 broken down by disease.

\section{Stratification of outcomes by diagnosis}

Figure 1 shows in detail what the outcomes were, based on the underlying disease and state. Early stages of diabetic eye affection (R1M0 and ROMO) had the highest discharge rate of all diabetic retinopathy stages. Patients graded as R1M1 had the highest rate of continuation in virtual clinic.

\section{Reasons for face to face clinic referral from the virtual clinic}

Table 3 gives the reasons stratified by disease why patients were brought to face to face clinic. The most common reason for a referral to a face-to-face clinic was poor image quality. This could be e.g. poor imaging acquisition technique or opacified optical media like advanced cataract.

\begin{tabular}{|l|l|l|l|l|}
\hline & Number & DNAs & Female & Age (years) \\
\hline Internal referrals & 1543 & $316(20.5 \%)$ & $701(45.4 \%)$ & 66.1 (female). 62.2 (male) \\
\hline New patients & 186 & $18(9.7 \%)$ & $85(45.7 \%)$ & 55.7 (female). 57.1 (male) \\
\hline
\end{tabular}

Table 1: Patient demographics of first time attendees to virtual clinic. DNA=patients that did not attend their appointment 


\begin{tabular}{|c|c|c|}
\hline & Internal referrals & New patients \\
\hline \multicolumn{3}{|l|}{ Patient data } \\
\hline Number of patients & 1227 & 168 \\
\hline Average review processing time & 8.34 days & 13.6 days \\
\hline VA of better eye (average) & 64.1 ETDRS letters & 81.5 ETDRS letters \\
\hline \multicolumn{3}{|l|}{ Disease and grade } \\
\hline Diabetic retinopathy (not graded) & $20(1.6 \%)$ & $2(1.2 \%)$ \\
\hline ROMO & $72(5.9 \%)$ & $2(1.2 \%)$ \\
\hline ROM1A & $1(0.1 \%)$ & \\
\hline R1M0 & $239(19.5 \%)$ & $38(22.8 \%)$ \\
\hline R1M1 & $351(28.6 \%)$ & $34(20.4 \%)$ \\
\hline R1M1A & $2(0.2 \%)$ & \\
\hline R1M1S & $39(3.2 \%)$ & \\
\hline R2MO & $32(2.6 \%)$ & $2(1.2 \%)$ \\
\hline R2M1 & $76(6.2 \%)$ & \\
\hline R2M1A & $8(0.7 \%)$ & \\
\hline R3MO & $56(0.7 \%)$ & \\
\hline R3M1 & $64(5.2 \%)$ & \\
\hline R3M1A & $7(0.6 \%)$ & \\
\hline Dry AMD & $74(6.0 \%)$ & $17(10.2 \%)$ \\
\hline Retinal vein occlusion & $56(4.6 \%)$ & $2(1.2 \%)$ \\
\hline Central serous retinopathy & $21(1.7 \%)$ & $2(1.2 \%)$ \\
\hline Other & $101(8.2 \%)$ & $68(40.7 \%)$ \\
\hline Not gradable due to bad image quality & $8(0.7 \%)$ & $1(0.6 \%)$ \\
\hline \multicolumn{3}{|l|}{ Outcomes } \\
\hline Percentage of patients discharged & $179(14.6 \%)$ & $76(45.5 \%)$ \\
\hline Percentage of patients continued in virtual clinic & $669(54.5 \%)$ & $62(37.1 \%)$ \\
\hline Time until next follow-up in virtual clinic & 211.6 days $(+/-80.3)$ & 243.8 days $(+/-78.8)$ \\
\hline Patients brought to face to face clinic & $379(30.9 \%)$ & $29(17.4 \%)$ \\
\hline Time until next follow-up in face to face clinic & 178.2 days $(+/-103.9)$ & 139.3 days $(+/-88.6)$ \\
\hline
\end{tabular}

\begin{tabular}{|c|c|c|c|c|c|c|c|c|}
\hline $\begin{array}{l}\frac{0}{0} \\
\frac{\pi}{60} \\
\frac{0}{0}\end{array}$ & 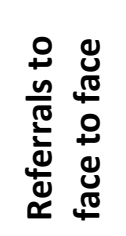 & 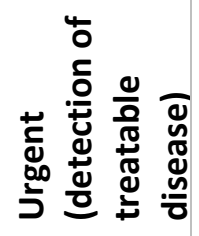 & 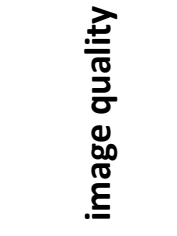 & 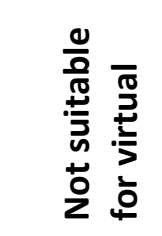 & 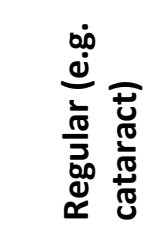 & 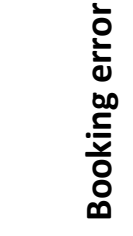 & 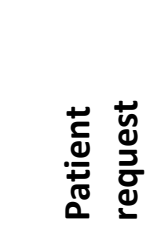 & 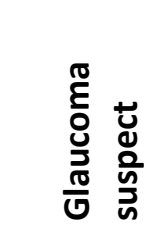 \\
\hline ROMO & 31 & $1(3.0 \%)$ & $19(57.6 \%)$ & $3(9.1 \%)$ & $8(24.2 \%)$ & & & \\
\hline R1M0 & 70 & $2(2.8 \%)$ & $45(63.4 \%)$ & $8(11.3 \%)$ & 12 (16.9\%) & $1(1.4 \%)$ & $1(1.4 \%)$ & $1(1.4 \%)$ \\
\hline R1M1 & 66 & 19 (28.4\%) & 12 (17.9\%) & 11 (16.4\%) & $22(32.8 \%)$ & $1(1.5 \%)$ & $1(1.5 \%)$ & \\
\hline R2MO & 14 & 1 (7.1\%) & $4(28.6 \%)$ & $4(28.6 \%)$ & $5(35.7 \%)$ & & & \\
\hline R2M1 & 36 & $14(37.8 \%)$ & $6(16.2 \%)$ & $9(24.3 \%)$ & $6(16.2 \%)$ & $1(2.7 \%)$ & & \\
\hline R3MO & 33 & $1(3.0 \%)$ & $11(33.3 \%)$ & $13(39.4 \%)$ & $6(18.2 \%)$ & $1(3.0 \%)$ & $1(3.0 \%)$ & \\
\hline R3M1 & 34 & $3(8.8 \%)$ & $14(41.2 \%)$ & $9(26.5 \%)$ & $7(20.6 \%)$ & & $1(2.9 \%)$ & \\
\hline AMD & 22 & $11(50 \%)$ & & $5(22.7 \%)$ & $5(22.7 \%)$ & $1(4.5 \%)$ & & \\
\hline RVO & 17 & $13(72.2 \%)$ & $1(5.6 \%)$ & $2(11.2 \%)$ & $1(5.6 \%)$ & & & \\
\hline CSR & 3 & $1(33.5)$ & & $2(66.7 \%)$ & & & & \\
\hline Total & 326 & 66 (20.2\%) & $112(34.7 \%)$ & 66 (20.2\%) & 72 (22.1\%) & $5(1.5 \%)$ & $4(1.2 \%)$ & $1(0.3 \%)$ \\
\hline
\end{tabular}




\begin{tabular}{|c|c|c|c|c|c|c|c|c|c|}
\hline \multirow{2}{*}{\multicolumn{2}{|c|}{ Disease and grade }} & \multicolumn{3}{|c|}{ Clinical rank } & \multirow{2}{*}{\multicolumn{2}{|c|}{ Disease and grade }} & \multicolumn{3}{|c|}{ Clinical rank } \\
\hline & & \multirow{2}{*}{$\begin{array}{l}\text { consultant } \\
20\end{array}$} & \multirow{2}{*}{$\begin{array}{l}\text { fellow } \\
1\end{array}$} & \multirow{2}{*}{$\begin{array}{l}\text { grader } \\
11\end{array}$} & & & \multirow{2}{*}{$\begin{array}{l}\text { consultant } \\
2\end{array}$} & \multirow[t]{2}{*}{ fellow } & \multirow{2}{*}{$\begin{array}{l}\text { grader } \\
1\end{array}$} \\
\hline RO & $\mathrm{F} 2 \mathrm{~F}$ & & & & CSR & $\mathrm{F} 2 \mathrm{~F}$ & & & \\
\hline & virtual & 12 & 0 & 5 & & virtual & 8 & & 4 \\
\hline & DC & 15 & 0 & 8 & & DC & 7 & & 0 \\
\hline \multirow[t]{3}{*}{$\mathbf{R 1}$} & $\mathrm{F} 2 \mathrm{~F}$ & 60 & 13 & 67 & \multirow[t]{3}{*}{ RVO } & $\mathrm{F} 2 \mathrm{~F}$ & 14 & 0 & 4 \\
\hline & virtual & 95 & 106 & 248 & & virtual & 24 & 1 & 3 \\
\hline & DC & 20 & 9 & 42 & & DC & 11 & 0 & 1 \\
\hline \multirow[t]{3}{*}{$\mathbf{R 2}$} & $\mathrm{F} 2 \mathrm{~F}$ & 18 & 9 & 23 & \multirow[t]{3}{*}{ AMD } & $\mathrm{F} 2 \mathrm{~F}$ & 15 & 2 & 5 \\
\hline & virtual & 15 & 9 & 34 & & virtual & 14 & 6 & 6 \\
\hline & DC & 0 & 0 & 1 & & DC & 38 & 0 & 5 \\
\hline \multirow[t]{3}{*}{ R3 } & $\mathrm{F} 2 \mathrm{~F}$ & 16 & 4 & 47 & & & & & \\
\hline & virtual & 21 & 8 & 21 & & & & & \\
\hline & $D C$ & 1 & 0 & 2 & & & & & \\
\hline
\end{tabular}

\section{Discussion}

In this study, the feasibility of implementing an integrated VMRC from four sites within a hospital eye service was demonstrated. In the 1,729 patients reviewed, the majority had a diagnosis of diabetic eye disease. We furthermore reviewed patients with other retinal conditions in the VMRC namely: AMD, RVO, and CSR. With an inclusion criteria set at the level where the legal requirements for driving are met, only a minority $0.7 \%$ of patients had ungradable images and therefore required an additional face-to-face clinic review. The turn-around time for obtaining an outcome in a virtual clinic was less than 2 weeks in most cases with a mean of 8 days for follow-up appointments. The majority of patients from internal referrals could be followed up in the virtual clinic whereas most of the new referrals could be discharged, creating capacity in the face-to-face medical retina clinics.

A major discussion point during the inception of these VMRCs was whether to implement "single-disease" clinics both at the point of image acquisition and outcome grading. A major advantage of "single-disease" clinics is the ability to train non-medical health professionals like in a DRSS. This means a significant difference in cost, as the price of one consultant's working hour is 41.11 pounds/hour compared to 17.26 pounds/hour of a grader. Overall, it could be shown in a systematic review study, that running a grader based screening service is cost-efficient and safe[21]. In this study, at most DR grades, the different clinical ranks decided similarly to either follow a patient up virtually, bring them to a face-to-face clinic or discharge them.

The advantage of "mixed-pathology" clinics is that it can free-up capacity in medical retina clinics, where there is a rising demand[7]. However, often patients do not need treatment like patients suffering from dry AMD and don't need to be followed up in a face-to-face clinic. This can be observed in our study, where a high percentage of patients with other diseases are being discharged. There is a tendency in consultants towards more decisive actions in all diseases, including diabetes. When running virtual clinics, an audit and feedback system could be implemented to assess quality and maintain a high and consistent standard of care.

To allow for further improvement of virtual clinics, referral criteria into virtual clinics allowing for good image quality should be well defined. We found that some referrals back to a faceto-face clinic might be avoidable by assessing clarity of optical media, as image quality already in the face-to-face clinic was low. In new referrals, this pre-selection is harder to make, as there is limited 
information about the patients from the DRSS. In a previous study, it could be shown, that age is the strongest predictor of ungradable image rates[22]. Non-mydriatic imaging is according to a systematic review inferior to dilated fundus exams[23]. In general, these referrals back into face-toface clinics cause duplication of work, reduction of efficiency, and increasing costs. We set the VA limit for new referrals into the virtual clinic at minimum legal driving requirements, as we expected to have patients with moderate to good optical media clarity and mild to moderate disease stages. This threshold could be modified and for example lowered where virtual clinics are the only way of delivering fast clinic appointments to the community (e.g. underserved areas, overburden of hospital eye services).

The nature of this study being retrospective is a limitation. However, it represents a largescale pilot project which contributes to help dealing with rising patient numbers and constraints in face-to-face appointments. Further studies need to be undertaken which analyse prospectively the outcome of this virtual medical retina service and demonstrate non-inferiority to today's gold standard, a clinical face to face eye examination. Also, the patients' perspectives were not reported, which was not in the scope of this paper. This may help to gain more insight, why patients did not attend (DNA) their appointment. From our experience, DNA rates in F2FC for referrals of R1M1 diabetic patients from DRSS are also between 15-25\%. More advanced DR states should be considered carefully for virtual review as i.e. no angle assessment can be done. Some patients with a more severe DR grade were seen in the virtual clinic, despite their DR grade being worse than defined in the referral criteria. This could be due to worsening of DR between appointments or due to under- or over grading of DR on examination; either in the F2FC or in the VMRC.

Although the introduction of VMRCs led to a drastic reduction of referral to appointment times, there is significant scope for further improving service access and reducing turnaround times. One reason for slow processing times might be improper software, which does not show all relevant clinical information along with images in one application. Currently, several software programs (EMR, worklist, OCT software, and fundus imaging software) need to be run in parallel. As they are not linked to each other, patient searches need to be carried out manually. A purpose-built software needs to be developed, ideally running in a secure cloud-based environment, enabling cross-site data entries and reviews. Also shared-care setting between eye hospitals and optometrists became possible. Centralised image grading could improve turnaround times further, as for this initial setup all reviews were done alongside the reviewers' daily work. A Reading Centre-based centralised review system utilising bespoke software, would drastically increase capacity for virtual clinics and enable economies of scale. -The emerging field of machine learning also holds promise for reducing processing times by analysing OCT scans and fundus images automatically, within a quality-assured failsafe structure with safety checks. Most of current approaches use deep-learning to achieve this task [24 25].

In this proof-of-concept report we demonstrate the potential of virtual clinics to enable rapid-access services for patients with medical retina low-risk referrals using up-to-date technology. Further adoption of modern technologies such as comprehensive virtual review platforms and machine learning tools along with the centralisation of the review process will further increase capacity while maintaining high standards of safety and quality of care. This could help to save limited resources for those who need it most. 


\section{Author's contributions}

Karsten Kortuem: Drafted the study, acquired, analysed and interpreted data, drafted the manuscript, gave final approval and is accountable for all aspects of the work

Katrin Fasler: Acquired data, revised the manuscript critically, gave final approval and is accountable for all aspects of the work

Amanda Charnley: Acquired data, revised the manuscript critically, gave final approval and is accountable for all aspects of the work

Hussain Khambati: Acquired data, revised the manuscript critically, gave final approval and is accountable for all aspects of the work

Sandro Fasolo: Build the database, revised the manuscript critically, gave final approval and is accountable for all aspects of the work

Menachem Katz: Build the database, revised the manuscript critically, gave final approval and is accountable for all aspects of the work

Konstantinos Balaskas: Drafted the study, revised the manuscript critically, gave final approval and is accountable for all aspects of the work

Ranjan Rajendram: Drafted the study, revised the manuscript critically, gave final approval and is accountable for all aspects of the work

Robin Hamilton: Drafted the study, revised the manuscript critically, gave final approval and is accountable for all aspects of the work

Pearse A. Keane: Drafted the study, revised the manuscript critically, gave final approval and is accountable for all aspects of the work

Dawn A. Sim: Drafted the study, acquired, analysed and interpreted data, drafted the manuscript, gave final approval and is accountable for all aspects of the work 
1. Field MJ. Telemedicine: A guide to assessing telecommunications for health care: National Academies Press, 1996.

2. Drexler W, Fujimoto JG. Optical coherence tomography: technology and applications: Springer, 2015.

3. Tham Y-C, Li X, Wong TY, Quigley HA, Aung T, Cheng C-Y. Global Prevalence of Glaucoma and Projections of Glaucoma Burden through 2040. Ophthalmology 2014;121(11):2081-90 doi: http://dx.doi.org/10.1016/j.ophtha.2014.05.013[published Online First: Epub Date]|.

4. Trikha S, Macgregor C, Jeffery M, Kirwan J. The Portsmouth-based glaucoma refinement scheme: a role for virtual clinics in the future? Eye (Lond) 2012;26(10):1288-94

5. Keenan J, Shahid H, Bourne RR, White AJ, Martin KR. Cambridge community Optometry Glaucoma Scheme. Clin Experiment Ophthalmol 2015;43(3):221-27

6. Kotecha A, Brookes J, Foster PJ. A technician-delivered 'virtual clinic' for triaging low-risk glaucoma referrals. Eye (Lond) 2017;31(6):899-905 doi: 10.1038/eye.2017.9[published Online First: Epub Date]|.

7. MacEwen C. Increasing demand on hospital eye services risks patients losing vision. Secondary Increasing demand on hospital eye services risks patients losing vision 2016. https://www.rcophth.ac.uk/2016/03/increasing-demand-on-hospital-eye-services-riskspatients-losing-vision/.

8. Resnikoff S, Felch W, Gauthier T-M, Spivey B. The number of ophthalmologists in practice and training worldwide: a growing gap despite more than 200000 practitioners. British Journal of Ophthalmology 2012;96(6):783-87 doi: 10.1136/bjophthalmol-2011-301378[published Online First: Epub Date]|.

9. Bellan L. Future trends in ophthalmology health human resources in Canada. Canadian Journal of Ophthalmology / Journal Canadien d'Ophtalmologie 2016;51(3):136-41 doi: http://dx.doi.org/10.1016/i.jcjo.2016.03.001[published Online First: Epub Date]|.

10. DaCosta J, Hamilton R, Nago J, et al. Implementation of a nurse-delivered intravitreal injection service. Eye (Lond) 2014;28(6):734-40 doi: 10.1038/eye.2014.69[published Online First: Epub Date]|.

11. Guariguata L, Whiting DR, Hambleton I, Beagley J, Linnenkamp U, Shaw JE. Global estimates of diabetes prevalence for 2013 and projections for 2035. Diabetes Research and Clinical Practice 2014;103(2):137-49 doi: http://dx.doi.org/10.1016/j.diabres.2013.11.002[published Online First: Epub Date]|.

12. Yau JWY, Rogers SL, Kawasaki R, et al. Global Prevalence and Major Risk Factors of Diabetic Retinopathy. Diabetes Care 2012;35(3):556-64 doi: 10.2337/dc11-1909[published Online First: Epub Date]|.

13. Stewart MW. Treatment of diabetic retinopathy: Recent advances and unresolved challenges. World Journal of Diabetes 2016;7(16):333-33 doi: 10.4239/wjd.v7.i16.333[published Online First: Epub Date]|.

14. Stein JD, Newman-Casey PA, Kim DD, Nwanyanwu KH, Johnson MW, Hutton DW. CostEffectiveness of Various Interventions for Newly Diagnosed Diabetic Macular Edema. Ophthalmology 2013;120(9):1835-42 doi:

http://dx.doi.org/10.1016/j.ophtha.2013.02.002[published Online First: Epub Date]|.

15. Olafsdottir E, Stefansson E. Biennial eye screening in patients with diabetes without retinopathy: 10-year experience. British journal of ophthalmology 2007;91(12):1599-601

16. Vijan S, Hofer TP, Hayward RA. Cost-utility analysis of screening intervals for diabetic retinopathy in patients with type 2 diabetes mellitus. Jama 2000;283(7):889-96

17. Liew $G$, Michaelides $M$, Bunce $C$. A comparison of the causes of blindness certifications in England and Wales in working age adults (16-64 years), 1999-2000 with 2009-2010. BMJ open 2014;4(2):e004015 
18. Manivannan A, Plskova J, Farrow A, McKay S, Sharp PF, Forrester JV. Ultra-Wide-Field Fluorescein Angiography of the Ocular Fundus. Am J Ophthalmol 2005;140(3):525-27 doi: http://dx.doi.org/10.1016/i.ajo.2005.02.055[published Online First: Epub Date]|.

19. Kiernan DF, Mieler WF, Hariprasad SM. Spectral-domain optical coherence tomography: a comparison of modern high-resolution retinal imaging systems. Am J Ophthalmol 2010;149(1):18-31. e2

20. Harding S, Greenwood R, Aldington S, et al. Grading and disease management in national screening for diabetic retinopathy in England and Wales. Diabetic medicine 2003;20(12):96571

21. Jones S, Edwards RT. Diabetic retinopathy screening: a systematic review of the economic evidence. Diabetic Medicine 2010;27(3):249-56 doi: 10.1111/j.14645491.2009.02870.x[published Online First: Epub Date]|.

22. Scanlon PH, Foy C, Malhotra R, Aldington SJ. The Influence of Age, Duration of Diabetes, Cataract, and Pupil Size on Image Quality in Digital Photographic Retinal Screening. Diabetes Care 2005;28(10):2448-53 doi: 10.2337/diacare.28.10.2448[published Online First: Epub Date]|.

23. Bedard C, Liu S, Patterson C, Gerstein H, Griffith L. Systematic review: Can non-mydriatic cameras accurately detect diabetic retinopathy? Diabetes Research and Clinical Practice 2017;129(Supplement C):154-59 doi: https://doi.org/10.1016/i.diabres.2017.04.024[published Online First: Epub Date]|.

24. Bogunovic $\mathrm{H}$, Montuoro A, Baratsits $\mathrm{M}$, et al. Machine Learning of the Progression of Intermediate Age-Related Macular Degeneration Based on OCT Imaging. Invest Ophthalmol Vis Sci 2017;58(6):Bio141-bio50 doi: 10.1167/iovs.17-21789[published Online First: Epub Date]|.

25. Gulshan V, Peng L, Coram M, et al. Development and validation of a deep learning algorithm for detection of diabetic retinopathy in retinal fundus photographs. JAMA 2016;316(22):240210 doi: 10.1001/jama.2016.17216[published Online First: Epub Date]|. 